\title{
O QUE A SISTEMATIZAÇÃO DA ASSISTÊNCIA DE ENFERMAGEM PODE OFERECER AO PACIENTE COM INFECÇÕES SEXUALMENTE TRANSMISSÍVEIS?
}

DOI: 10.48140/digitaleditora.2021.005.7

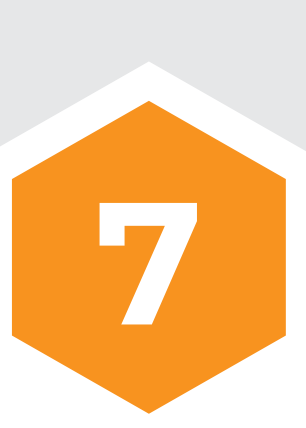

RESUMO

Objetivos: Elaborar um plano de cuidados em enfermagem a pacientes com infecções sexualmente transmissíveis.

Metodologia: Trata-se de uma revisão integrativa da literatura, que é um método de pesquisa que realiza a busca, a avaliação crítica e a síntese de estudos publicados sobre um determinado tema de forma sistemática.

Resultados: O instrumento foi composto por 5 fases, estas são organizadas de forma estratégica, com os diagnósticos de enfermagem, distúrbio na imagem corporal, risco de infecção, estilo de vida sedentário, baixa autoestima situacional, isolamento social e padrão de sexualidade ineficaz, com as intervenções dadas pela 'Classificação das Intervenções de Enfermagem (NIC)' para as Necessidades Humanas Básicas afetadas dos pacientes, sendo elas, conforto, sexualidade, segurança, autoimagem, proteção e autopercepção.

Discussão: A SAE é uma metodologia científica que vem sendo cada vez mais implementada na prática assistencial e é um excelente instrumento para realização do cuidado.

Conclusão: Ficou evidente que a importância da consulta de enfermagem ao paciente com infecções sexualmente transmissíveis pode trazer inúmeros benefícios tanto individuais como coletivos, através da anamnese e exame físico é possível traçar os diagnósticos, estabelecer os resultados e propor as intervenções ou plano de cuidados a esses pacientes.

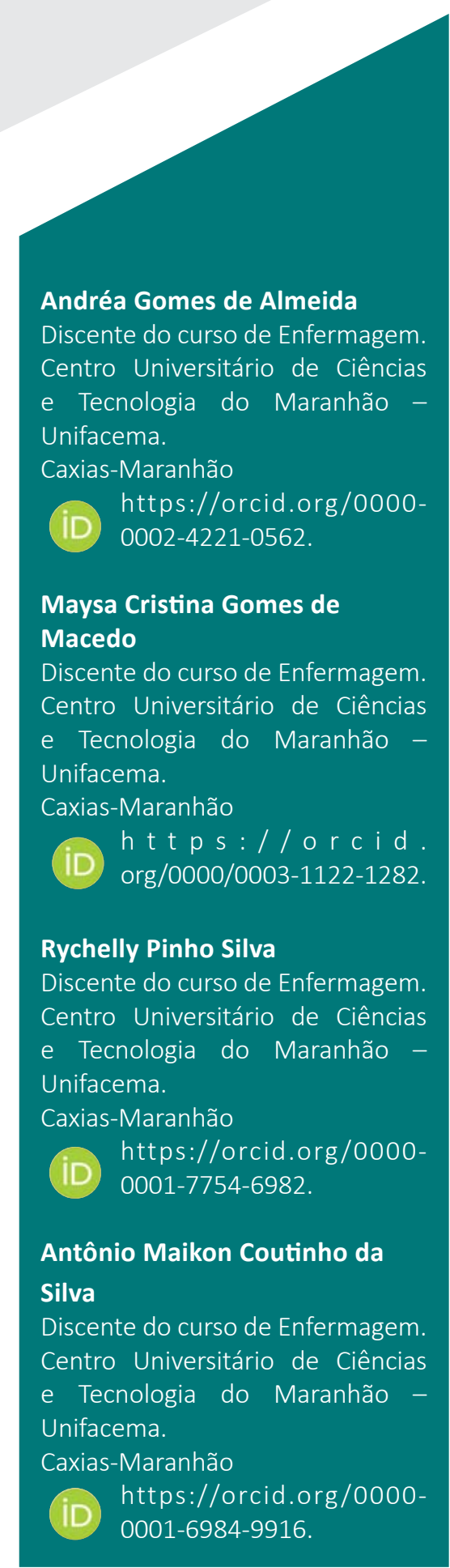

PALAVRAS-CHAVES: Infecções sexualmente transmissíveis; Enfermagem; Sistematização da Assistência de Enfermagem. 


\section{WHAT CAN THE SYSTEMATIZATION OF NURSING CARE OFFER PATIENTS WITH SEXUALLY TRANSMITTED INFECTIONS?}

DOI: 10.48140/digitaleditora.2021.005.7

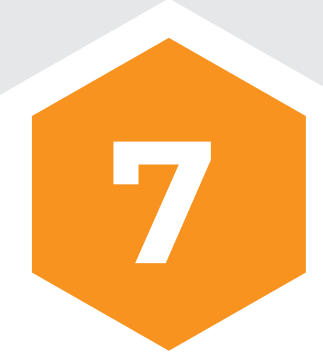

ABSTRACT

Recebido em: 30/11/2020

Aprovado em: 10/12/2020

Conflito de Interesse: não

Suporte Financeiro: não houve
Objectives: Objectives: Develop a nursing care plan for patients with sexually transmitted infections. Methods: This is an integrative review of the literature, which is a research method that performs the search, critical evaluation and synthesis of published studies on a given theme systematically.

Results: The instrument was composed of 5 phases, these are organized strategically, with nursing diagnoses, body image disorder, risk of infection, sedentary lifestyle, low situational selfesteem, social isolation and pattern of ineffective sexuality, with the interventions given by the 'Classification of Nursing Interventions (NIC)' for the Affected Basic Human Needs of patients, , they are comfort, sexuality, safety, self-image, protection and self-perception. Discussion: SAE is a scientific methodology that has been increasingly implemented in care practice and is an excellent instrument for performing care.

Conclusion: It was evident that the importance of nursing consultation with patients with sexually transmitted infections can bring numerous benefits both individual and collective, through anamnesis and physical examination it is possible to trace the diagnoses, establish the results and propose interventions or care plan to these patients. 


\section{INTRODUÇÃO}

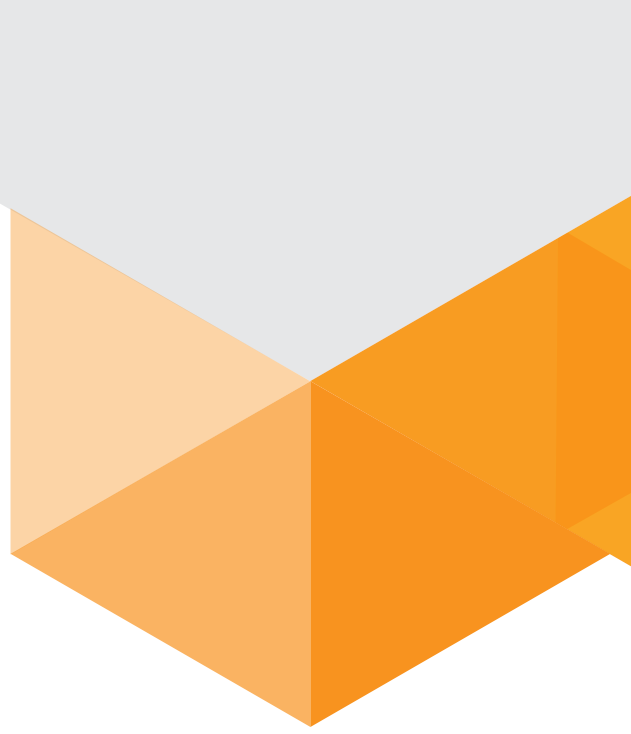

A Sistematização da Assistência de Enfermagem (SAE) baseia-se na estruturação da forma de tratar o sujeito, por meio de um olhar holístico, fazendo valer o princípio da integralidade, com ênfase não só nas necessidades biológicas, mas nas emocionais, psicológicas, sociais e espirituais. Esse método requer do profissional de enfermagem um conhecimento científico e técnico para o delineamento da abordagem ao sujeito e os passos seguintes: formulação e concretização das etapas de cada fase do processo. Este profissional tem autonomia para executar seu senso crítico diante de diversas situações no contexto da saúde (GARCIA; NÓBREGA, 2009).

A Sistematização da Assistência à Enfermagem (SAE) é entendida como qualquer conteúdo/ação que organize o trabalho profissional do enfermeiro, com base teórico-filosófica, que permita a operacionalização do Processo de Enfermagem (PE), com base teórico-filosófica (SILVA, 2017). Logo o processo de enfermagem é a ferramenta que operacionaliza a SAE. O processo de Enfermagem possibilita ao enfermeiro desenvolver e aplicar a SAE através do conhecimento técnico-científico, de modo a manter mesmo que mínimo, o nível de saúde do paciente, pois visa planejar, executar e avaliar o cuidado, tratando-se de ferramenta fundamental no trabalho do enfermeiro ao paciente, nesse caso com IST's (HORTA, 1079).

O "autocuidado inclui ações deliberadas e sistematizadas, desempenhadas para tornar conhecidas necessidades de cuidado; "O autocuidado promove o desenvolvimento dos seres humanos" (LEOPARDI, 1999 p.76). E dando continuidade ao processo de enfermagem, chegou o momento de se escolher qual sistema de classificação de enfermagem que você enfermeiro irá utilizar para programar o SAE com o seu paciente/cliente. Sendo assim vale ressaltar que existem vários sistemas de classificação de enfermagem. No Brasil, os mais conhecidos e utilizados são: classificação diagnóstica da NANDA-I (North American Nursing Diagnoses Association), classificação das intervenções de enfermagem NIC (Nursing Intervention Classification), classificação dos resultados de enfermagem NOC (Nursing Outcome Classification), e a CIPE $^{\circledR}$ (Classificação Internacional para a Prática de Enfermagem) (MATA; SOUZA; CHIANCA et al., 2012). Deste modo, o sistema de classificação que será utilizado neste estudo será o sistema NNN, que compreender; NANDA, NIC e NOC, para a elaboração dos diagnósticos de enfermagem, assim como, os resultados e intervenções de enfermagem.

As Infeç̧ões Sexualmente Transmissíveis (IST) são problemas de Saúde Pública, devido à sua magnitude e dificuldade de acesso ao tratamento adequado. Em 2012 a incidência global foi estimada em 
357,4 milhões de casos novos de IST curáveis no mundo, na faixa etária de 15 a 49 anos, a maioria deles em países em desenvolvimento (NEWMAN et al., 2015).

A Organização Mundial de Saúde (OMS) estima que mais de um milhão de pessoas adquirem IST diariamente no mundo, sendo 500 milhões com IST curáveis, outros 530 milhões contaminados com o vírus do herpes genital e cerca de 290 milhões de mulheres com Papiloma Vírus Humano (BRASIL, 2015a).

Para combater as IST, é importante a realização de ações de enfermagem que interrompam a cadeia de transmissão, detectando precocemente os casos, tratando os infectados e seus parceiros, prevenindo-os de outras IST e de complicações advindas das infecções, a partir do aconselhamento na consulta de enfermagem (BRASIL, 2015b).

Segundo a Portaria № 1.625 de 10 de julho de 2007, é de incumbência do enfermeiro da Equipe de Saúde da Família (ESF) realizar consultas de enfermagem (BRASIL, 2007). A consulta de enfermagem tem como objetivo o diagnóstico preciso e a elaboração de um plano de cuidados, de acordo com a necessidade de cada paciente para obtenção de um bom resultado (MARTINS; FERRAZ, 2014).

Tem-se ainda na literatura poucos estudos que mostra uma aplicação direta da SAE e processo de enfermagem especificamente a esse grupo de pessoas com infecções sexualmente transmissíveis. $O$ principal objetivo deste estudo foi elaborar um plano de cuidados em enfermagem a pacientes com infecções sexualmente transmissíveis com estabelecimento de condutas de enfermagem, com a aplicação do processo de enfermagem (PE) e a sistematização da assistência de enfermagem (SAE).

\section{METODOLOGIA} de estudos publicados sobre um determinado tema de forma sistemática. As revisões integrativas também apontam lacunas do conhecimento que necessitam ser preenchidas com a realização de novos estudos (MENDES; SILVEIRA; GALVÃO, 2008).

Nossas buscas foram realizadas nas seguintes bases de dados: $\mathrm{Bi}-$ blioteca Científica Eletrônica Online (SCIELO), BIREME- Centro Latino Americano e do Caribe de Informação em Ciências da Saúde, MEDLINE- Sistema Online de Busca e Análise de Literatura Médica,

Quando realizadas as buscas no Pub Med/MEDILINE, foram utilizados os filtros - texto completo, ensaio clínico, ensaio clínico randomizado, revisão, revisão sistemática, últimos 5 anos, com 18,308 resultados encontrados, destes somente seis (6) foram lidos, pois se encaixam nos objetivos da pesquisa. Usando os termos "systematization of nursing care".

$\mathrm{Na}$ base de dados Scielo, sem filtros, tiverem 167 artigos como resultados, destes, 3 foram utilizados. Usando os termos "sistematização da assistência de enfermagem".

E na BIREME, os resultados com os filtros: texto completo, MEDILINE, LILACS, BDENF, doenças sexualmente transmissíveis, estudo de prevalência, estudo de avaliação, relato de caso e revisão sistemática, com os idiomas português e inglês. Usando os termos "infecções sexualmente transmissíveis", obtendo 806 artigos, onde 4 foram analisados. 


\section{RESULTADOS}

PROCESSO DE ENFERMAGEM - O instrumento é composto por 5 fases, estas são organizadas de forma estratégica. São elas:

Investigação ou Histórico de Enfermagem - (Anamnese e Exame Físico) - discute a respeito da investigação para identificar problemas e necessidades do paciente e assim, determinar seu estado de saúde. Descreve cinco passos que auxiliam e ordena o profissional enfermeiro: a coleta de dados, validação dos dados, agrupamento dos dados, identificação de padrões e comunicação e registro dos dados. Conforme quadro 1 abaixo.

Quadro 1: Instrumento de entrevista. Histórico de Enfermagem ao paciente com IST's.

\section{SAE NA PRIMEIRA CONSULTA AO USUÁRIO COM IST IDENTIFICAÇÃO DO PACIENTE}

\section{IDENTIFICAÇÃO DO PACIENTE}

Nome: Data Nasc: Car-

tão do SUS:

Est. Civil: ( ) solteiro ( ) casado/amas. ( ) separado ( ) viúvo Orientação sexual: ( ) F ( ) M ( ) Travesti ( ) Transgênero Naturalidade: Residência: Tempo de residência: Telefone: ( ) | Gestante: ( )

Sim ( ) Não ( ) Não se aplica Etnia/cor: ( ) 1-Branca; 2-Preta; 3-Amarela; 4-Parda; 5-Indígena; 9-Ignorado Profissão: ( ) Desempregado | ( ) Benefício Renda mensal: $\mathrm{R} \$$ Escolaridade: Deficiente: ( ) Visual ( ) Auditivo ( ) Físico Contato particular ou pessoal, caso necessário: DIAGNÓSTICO inicial: Data do diagnóstico:

_____ Faz uso de medicamentos: ( ) Não ( \} Sim Quais: Data do início do tratamento medicamentoso: Como foi feito o diagnóstico: ( ) Busca ativa ( ) Solicitação médica ( ) Banco de sangue ( ) CTA ( ) Pronto atendimento ( ) Atenção básica ( ) Atenção terciária Outro: ANTECEDENTES PESSOAIS: Orientação sexual: ( ) Heterossexual ( ) Homossexual ( ) Bissexual Parceria Sexual: ( ) Homem ( ) Mulher ( ) Travesti/Transexual ( ) Não informado ( ) Parceiro fixo ( ) Parceiro eventual Tempo de relacionamento: $\mathrm{N}^{\circ}$ de parceiros:

Uso de preservativo: ( ) sempre ( ) Às vezes ( ) Nunca Teve alguma DST nos últimos 12 meses? ( ) Não ( ) Sim, qual? Uso de substância química: ( ) Álcool ( ) Tabaco ( ) Maconha ( ) Cocaína ( ) Crack ( ) Anfetaminas ( ) Drogas ilícitas injetáveis ( ) Drogas ilícitas inaláveis ( ) Outros: Tempo de uso: Frequência: Presidiário ou Ex-presidiário: ( ) Não ( ) Sim - Perío-

do: de a Compartilha ou já compartilhou: ( ) Alicate de unha ( ) Gillette ( ) Aparelho de barbear ( ) Escova de dentes ( ) Seringas e Agulhas ( ) Canudo ou cachimbo (uso de drogas) Você tem kit individual para fazer unha? ( ) Sim ( ) Não ( ) Não faço unha Apresenta tatuagem: ( ) Sim ( ) Não Piercing: ( ) Sim ( ) Não Já recebeu transfusão de sangue/transplante de órgãos? ( ) Não ( ) Sim, quando? Vacinação para Hepatite B: ( ) Nenhuma dose ( ) 1 dose ( ) 2 doses ( ) 3 doses ( ) doses Comorbidades: ( ) HAS ( ) DM ( ) Cardiopatias ( )Dislipidemias ( ) Neoplasias ( ) HTLV ( ) Lipodistrofia ( ) Hepatopatias ( ) Neuropatias outras: Coinfecção: ( ) TB ( ) Pneumonia ( ) 
Toxoplasmose ( ) Sífilis ( ) Outras DSTs: do: de a naturais? ( ) Sim ( ) Não Quais?

I

Tratamento anterior por TB: ( ) Não ( ) Sim - PeríoFaz uso de anticoncepcionais? ( ) Não ( ) Sim, qual Tratamentos Medicamentos em uso: Convive ou conviveu com doentes de Tuberculose? ( ) Sim ( ) Não Parentesco: AVALIAÇÃO

( ) Não sabe Se sim, nome: DIAGNÓSTICA INICIAL: ( ) TMR-TB: ( ) Positivo ( ) Negativo ( ) Baciloscopia: ( ) Positiva ( ) Negativa ( ) Cultura de Escarro: ( ) Positiva ( ) Negativa ( ) PPD: ( ) Anti-HIV: ( ) Reagente ( ) Não Reagente ( ) Anti HCV: ( ) Reagente ( ) Não Reagente ( ) HBsAg: ( ) Reagente ( ) Não Reagente ( )Anti Hbc total: ( ) Reagente ( )Não Reagente ( ) Anti HBs: ( ) Reagente ( ) Não Reagente ( ) Sífilis TP: ( ) Reagente ( ) Não Reagente ( ) VDRL: I I Outros: EXAME FÍSICO GERAL CABEÇA E PESCOÇO

Avaliação Neurológica: (Nível de consciência) ( )sonolento ( )púpilas isocóricas ( )força motora presente ( ) força motora ausente ( )sensibilidade tátil presente ( )sensibilidade tátil ausente ( )função motora preservada ( )função motora ausente Avaliação dos pares de Nervos Cranianos (Sistema Nervoso Periférico (SNP): Nervo Olfatório: ( )Hiposmia (baixa sensibilidade auditiva) ( )Hipersensibilidade auditiva Nervo Óptico: ( ) acuidade visual preservada( ) acuidade visual diminuida, Nervo Oculomotor: Troclear e Abducente: ( )movimento dos olhos normais ( )movimento dos olhos anormais ( ) reflexo corneopalpebral presente ( )reflexo corneopalpebral ausente. Cabeça: ( )Simétrica ( )Assimétrica ( )Alterações.Quais: Couro cabeludo: ( ) Integro ( )Alterações ( )Boas condições de higiene ( )Más condições de higiene Pupilas: ( )Isocóricas(fotoreagentes) ( )midriáticas ( )mióticas ( )anisocóricas ( )discórica Nariz e boca: ( )integra ( )alterações. Quais: Prótese dentária: ( ) sim ( ) não Fala/linguagem: ( )sem alteração ( ) com alteração

\section{TÓRAX}

Padrão respiratório: ( )eupnéico ( )dispneico ( )taquipnéico ( )apneia ( )bradipneico ( )ortopneico ( ) Kussmaul ( ) Cheyne-Stokes Oxigenioterapia: ( )cateter de O2 ( )macronebulização ( )venturi ( )máscara de reservatório Ventilação mecânica: ( )TOT ( )TQT Ausc.Pulmonar: ( )MV+ ( )MV diminuídos ( )sem RA ( ) com RA: ( )creptantes ( )roncos ( )sibilos ( )estertores bolhosos ( )atrito pleural ( )cornagem/estridor Ausc. Cardiovascular: ( )BRNF 2T ( )s/ sopro ( ) c/ sopro ( ) Outros:

\section{ABDOME}

Contorno do abdome: ( )plano ( )escavado ( )distendido ( )globoso ( )flácido ( )avental ( )protuberante ( ) timpânico ( )maciço ( )submaciço ( )doloroso ( )indolor Parede abdominal: ( )integra ( ) presença de cicatrizes ( )estrias Ruídos intestinais: ( )RHA + ( )RHA - ( )RHA ausente ( )normoativos ( )hipoativos ( )hiperativos Hérnia: ( ) sim ( ) não Obs.:

\section{APARELHO GENITURINÁRIOÂNUS E RETO}

Micção: ( )espontânea ( )disúria ( )anúria ( )oligúria ( )poliúria ( )nictúria ( )SVD ( )perda urinária Teste: *Giordano: ( ) + / ( ) - Método: *DEVOTO ( ) + / ( ) - * ISRAEL( ) + / ( ) - Aspecto: ( )inalterado ( )hematúria ( )polaciúria ( ) lesões ( )varizes vulvares ( )edema ( )períneo integro ( )EMLD ( )hemorróidas Obs.:

Diurese: ( ) Presente ( )Ausente Obs.: MMSS E MMII Mobilidade

Física: ( )deambula ( ) acamado ( )restrito ao leito Uso de prótese: ( )sim ( )não / localização: MMSS: ( ) dor edema ( ) não ( ) sim ( /4+) ( )AVP/ local ( ) CVC/local MMII: ( ) dor edema ( ) não ( ) sim ( /4+) ( )Varizes ( hematoma/Local: Alterações: ( ) Outros: PELEEANEXOS Pele: ( )integra ( )cicatriz ( )coloração:

Perfusão Periférica: ( )boa ( )ruim ( )regular Hematoma/local: Mucosas: ( )coradas ( )descoradas + $14+$ OBS:

Data:_ I_ I_ HORA:

ASSINATURA DO(A) ENFERMEIRO(A) CARIMBO :

Fonte: Elaboração do autor, 2020. 
Diagnósticos de Enfermagem - descreve a evolução histórica de como os diagnósticos foram elaborados e desenvolvidos por enfermeiros. Também aborda os treze domínios utilizados no sistema de classificação NANDA e também os componentes estruturais que devem conter neste sistema;

Planejamento dos Resultados Esperados - foca na importância de realizar o planejamento de enfermagem após elaboração do diagnóstico. Através dos resultados esperados estabelecidos são realizadas prescrições de enfermagem para que a meta proposta seja alcançada;

Implementação da Assistência de Enfermagem (Prescrição de Enfermagem) - é descrito passos para realizar uma prescrição de enfermagem e implementá-la a fim de atingir a meta proposta. Para as autoras, as prescrições de cuidados devem estar bem redigidas e despertar o interesse da equipe de enfermagem, tanto para ler quanto para realizar. Aplicadas essas fases aqui abaixo, conforme quadro 2.

Quadro 2: Diagnósticos de Enfermagem, paciente portador de Infecções Sexualmente Transmissíveis (IST's).

\begin{tabular}{|c|c|c|c|c|c|}
\hline \multicolumn{6}{|c|}{ Diagnósticos de Enfermagem } \\
\hline N.H.B & $\begin{array}{l}\text { Diagnóstico } \\
\text { de Enferma- } \\
\text { gem (NAN- } \\
\text { DA-I) }\end{array}$ & $\begin{array}{l}\text { Fator Relacio- } \\
\text { nado }\end{array}$ & $\begin{array}{l}\text { Caract. Defi- } \\
\text { nidoras }\end{array}$ & $\begin{array}{l}\text { Resultados } \\
\text { Esperados }\end{array}$ & Sugestões de Intervenções \\
\hline $\begin{array}{l}\text { Autoi- } \\
\text { magem }\end{array}$ & $\begin{array}{l}\text { Distúrbio } \\
\text { na Imagem } \\
\text { Corporal }\end{array}$ & $\begin{array}{l}\text { - Alteração na } \\
\text { autopercep- } \\
\text { ção. } \\
\text { - Doença } \\
\text { (IST's). } \\
\text { - Tratamento. }\end{array}$ & $\begin{array}{l}\text { - Alteração } \\
\text { na estru- } \\
\text { tura cor- } \\
\text { poral } \\
\text { - Alteração } \\
\text { na visão } \\
\text { do próprio } \\
\text { corpo } \\
\text { - Esconder } \\
\text { parte do } \\
\text { corpo } \\
\text { - Medo da } \\
\text { reação dos } \\
\text { outros }\end{array}$ & $\begin{array}{l}\text { Satisfação } \\
\text { com a função } \\
\text { corporal }\end{array}$ & $\begin{array}{l}\text { - Usar orientação antecipada } \\
\text { para preparar o paciente para } \\
\text { mudanças previsíveis na ima- } \\
\text { gem corporal. } \\
\text { - Determinar se o desgosto per- } \\
\text { cebido em relação a algumas } \\
\text { características físicas cria pa- } \\
\text { ralisia social disfuncional em } \\
\text { adolescentes e outros grupos } \\
\text { de alto risco. } \\
\text { - Ajudar o paciente a discutir } \\
\text { mudanças causadas por do- } \\
\text { ença ou cirurgia, conforme } \\
\text { apropriado. } \\
\text { - Ajudar o paciente a determi- } \\
\text { nar o alcance das mudanças } \\
\text { reais no corpo ou nível de fun- } \\
\text { cionamento. } \\
\text { - Determinar se alguma mu- } \\
\text { dança física recente foi incor- } \\
\text { porada à imagem corporal do } \\
\text { paciente. } \\
\text { - Ajudar o paciente a separar } \\
\text { aparência física de sentimen- } \\
\text { tos de valor pessoal, conforme } \\
\text { apropriado. } \\
\text { - Ajudar o paciente a determi- } \\
\text { nar a influência do grupo de } \\
\text { colegas na percepção do pa- } \\
\text { ciente sobre a imagem corpo- } \\
\text { ral atual. } \\
\text { declarações de autocrítica. }\end{array}$ \\
\hline
\end{tabular}




\begin{tabular}{|c|c|c|c|c|c|}
\hline $\begin{array}{l}\text { Segu- } \\
\text { rança/ } \\
\text { proteção }\end{array}$ & $\begin{array}{l}\text { Risco de } \\
\text { Infecção }\end{array}$ & $\begin{array}{l}\text { - Alteração na } \\
\text { integridade } \\
\text { da pele. } \\
\text { - Conheci- } \\
\text { mento in- } \\
\text { suficiente } \\
\text { para evitar } \\
\text { exposição a } \\
\text { patógenos. } \\
\text { - Vacinação } \\
\text { inadequada. }\end{array}$ & $X$ & $\begin{array}{l}\text { Detecção de } \\
\text { Riscos }\end{array}$ & $\begin{array}{l}\text { - Monitorar a continuidade ade- } \\
\text { quadas vacinas na população. } \\
\text { - Promover o acesso à educa- } \\
\text { ção à saúde coletiva e preven- } \\
\text { ção de doenças transmissíveis. } \\
\text { - Monitorar os fatores ambien- } \\
\text { tais que influenciam a trans- } \\
\text { missão de doenças contagio- } \\
\text { sas (IST's). } \\
\text { - Monitorar as medidas sanitá- } \\
\text { rias. }\end{array}$ \\
\hline $\begin{array}{l}\text { Promo- } \\
\text { ção da } \\
\text { saúde. }\end{array}$ & $\begin{array}{l}\text { Estilo de } \\
\text { vida seden- } \\
\text { tário }\end{array}$ & $\begin{array}{l}\text { Conhecimen- } \\
\text { to insuficiente } \\
\text { sobre os bene- } \\
\text { fícios à saúde } \\
\text { associados } \\
\text { ao exercício } \\
\text { físico Interes- } \\
\text { se insuficiente } \\
\text { em atividades } \\
\text { físicas. }\end{array}$ & $\begin{array}{l}\text { Falta de } \\
\text { condicio- } \\
\text { namento } \\
\text { físico Média } \\
\text { de atividade } \\
\text { física diária } \\
\text { inferior à re- } \\
\text { comendada } \\
\text { para idade e } \\
\text { sexo Prefe- } \\
\text { rência por } \\
\text { atividades } \\
\text { com pouca } \\
\text { atividade } \\
\text { física }\end{array}$ & $\begin{array}{l}\text { Aptidão } \\
\text { Física }\end{array}$ & $\begin{array}{l}\text { Avaliar as crenças de saúde do } \\
\text { indivíduo sobre exercícios físi- } \\
\text { cos. Investigar experiências an- } \\
\text { teriores com exercícios. Deter- } \\
\text { minar a motivação do indivíduo } \\
\text { para começar/continuar um } \\
\text { programa de exercícios. Inves- } \\
\text { tigar as barreiras ao exercício. } \\
\text { Encorajar a expressão de sen- } \\
\text { timentos sobre o exercício ou a } \\
\text { necessidade dele. Encorajar a } \\
\text { pessoa a começar ou continuar } \\
\text { os exercícios. Auxiliar na ident- } \\
\text { ficação de um modelo de papel } \\
\text { positivo para manter o progra- } \\
\text { ma de exercícios. Auxiliar o indi- } \\
\text { víduo a desenvolver um progra- } \\
\text { ma adequado de exercícios que } \\
\text { atenda às suas necessidades. } \\
\text { Auxiliar a pessoa a estabelecer } \\
\text { metas de curto e longo prazos } \\
\text { para o programa de exercícios. }\end{array}$ \\
\hline $\begin{array}{l}\text { Autoper- } \\
\text { cepção }\end{array}$ & $\begin{array}{l}\text { Baixa au- } \\
\text { toestima } \\
\text { situacional. }\end{array}$ & $\begin{array}{l}\text { Alteração da } \\
\text { imagem cor- } \\
\text { poral Altera- } \\
\text { ção no papel } \\
\text { social } \\
\text { Autoexpec- } \\
\text { tativas não } \\
\text { realistas Com- } \\
\text { portamento } \\
\text { inconsistente } \\
\text { em relação } \\
\text { aos valores. }\end{array}$ & $\begin{array}{l}\text { Desafio si- } \\
\text { tuacional ao } \\
\text { próprio valor } \\
\text { Desamparo } \\
\text { Subestima a } \\
\text { capacidade } \\
\text { de lidar com } \\
\text { a situação } \\
\text { Verbaliza- } \\
\text { ções auto- } \\
\text { negativas }\end{array}$ & $\begin{array}{l}\text { Resiliência } \\
\text { Pessoal }\end{array}$ & $\begin{array}{l}\text { Monitorar as declarações de } \\
\text { autovalorização do paciente. } \\
\text { Determinar o lócus de contro- } \\
\text { le do paciente. Determinar a } \\
\text { confiança do paciente no pró- } \\
\text { prio julgamento. Encorajar o } \\
\text { paciente a identificar os pontos } \\
\text { fortes. Encorajar o contato com } \\
\text { os olhos na comunicação com } \\
\text { os outros. Reforçar os pontos } \\
\text { positivos pessoais identificados } \\
\text { pelo paciente. Proporcionar ex- } \\
\text { periências que aumentem a au- } \\
\text { tonomia do paciente, conforme } \\
\text { apropriado. Ajudar o paciente a } \\
\text { identificar reações positivas dos } \\
\text { outros. Evitar críticas negativas. }\end{array}$ \\
\hline
\end{tabular}




\begin{tabular}{|c|c|c|}
\hline Conforto & $\begin{array}{l}\text { Isolamento } \\
\text { social }\end{array}$ & $\begin{array}{l}\text { Dificuldade } \\
\text { para estabele- } \\
\text { cer relaciona- } \\
\text { mentos Inca- } \\
\text { pacidade de } \\
\text { engajar-se em } \\
\text { relacionamen- } \\
\text { tos pessoais } \\
\text { satisfatórios } \\
\text { Interesses } \\
\text { inadequados } \\
\text { para o nível } \\
\text { de desenvolvi- } \\
\text { mento }\end{array}$ \\
\hline $\begin{array}{l}\text { Sexuali- } \\
\text { dade }\end{array}$ & $\begin{array}{l}\text { Padrão de } \\
\text { sexualidade } \\
\text { ineficaz }\end{array}$ & $\begin{array}{l}\text { Medo de } \\
\text { infecção } \\
\text { sexualmente } \\
\text { transmissível } \\
\text { Relaciona- } \\
\text { mento preju- } \\
\text { dicado com } \\
\text { uma pessoa } \\
\text { significativa. }\end{array}$ \\
\hline
\end{tabular}

Dificuldade para estabeleer relaciona-

Insegurança em público

Afeto

superficial

Afeto triste

Ausência de

propósito

Alteração

na ativida-

de sexual

Alteração no

comporta-

al Alteração Sexual

no relacio-

namento

com pessoa

significativa
Encorajar melhoria do envolvimento em relações já criadas. Encorajar a paciência no desenvolvimento de relações. Promover relações com pessoas com interesses e metas comuns. Encorajar atividades sociais e comunitárias. Promover o compartilhamento de problemas comuns com os outros. Encorajar a honestidade ao apresentar-se aos outros. Promover o envolvimento em interesses completamente novos. Encorajar o respeito aos direitos dos outros.

Oferecer educação sexual;

Proporcionar aconselhamento;

Estimular o relacionamento como forma de apoio e companheirismo;

Discutir com o paciente as consequencias de comportamento sexual e verbalizações socialmente inaceitáveis.

Fonte: NANDA, NIC e NOC. Quadro elaboração do autor, 2020.

Avaliação da Assistência de Enfermagem - aborda a etapa que consiste em acompanhar as respostas do paciente aos cuidados e avaliar se obteve bons resultados das prescrições de enfermagem. Deve ser realizado diariamente ou a cada novo contato com o paciente durante o procedimento do exame físico.

\section{DISCUSSÃO}

A SAE é uma metodologia científica que vem sendo cada vez mais implementada na prática assistencial e é um excelente instrumento para realização do cuidado, recuperação e aumentar a qualidade de vida do paciente, também proporcionando um cuidado de enfermagem individualizado, contínuo, seguro e pautado em princípios científicos, aumentando o prognóstico de vida dos pacientes.

O processo de enfermagem é um método para implantar, na prática profissional uma teoria de enfermagem. Como falaremos de prevenção e promoção da saúde, a de se pensar em educação em saúde que é considerada uma ação básica que tem como objetivo capacitar indivíduos e grupos para seu autocuidado na saúde, oportunizando mudanças de comportamentos, práticas e atitudes para aquisição de melhores condições de vida, ainda mais se tratando de orientação Sexual e IST (CARVALHO; FREITAS; SOUZA et al., 2014). 
É dever do enfermeiro orientar as usuárias do serviço quanto a multiplicidade de parceiros, a importância do uso da camisinha como dupla proteção, a presença de vulvovaginites, importância do tratamento do parceiro para interromper a cadeia de transmissão das IST e sobre os fatores de risco para contaminação do HPV e suas principais consequências, como o câncer do colo do útero, embasando a importância da realização periódica do exame preventivo e a volta à unidade de saúde para buscar o resultado do exame citopatológico (OLIVEIRA; LIMA; CAMPOS et al., 2013a). A OMS, devido à transcendência das IST, apresenta, periodicamente, estimativas da magnitude destas infecções no mundo, para subsidiar a implementação de políticas públicas para seu controle (OMS, 2012)

Outra importante estratégia de prevenção para todas as IST é a divulgação sobre as formas de transmissão, os sinais e os sintomas de IST (ALVES; KOVACS; STALL et al., 2002). Visto que o comportamento sexual de risco é caracterizado por iniciação sexual precoce, multiplicidade de parceiros, a ausência de preservativo nas relações sexuais, utilização de álcool e substancias psicoativas, aspectos importantes que deveriam ser avaliados durante a consulta de enfermagem (OLIVEIRA; LIMA; CAMPOS et al., 2013b). 


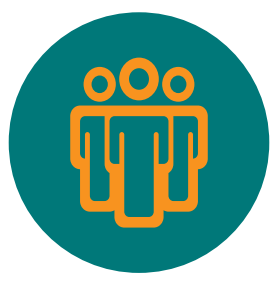

\section{CONCLUSÃO}

No presente estudo, ficou evidente que a importância da consulta de enfermagem ao paciente com infecções sexualmente transmissíveis pode trazer inúmeros benefícios tanto individuais como coletivos, visto que a sistematização da assistência de enfermagem junto ao processo de enfermagem se configura como fortes estratégias para o contexto caótico das IST's.

Os enfermeiros possuem papel fundamental no estabelecimento das condutas preconizadas pelo Ministério da Saúde para consulta de enfermagem. Através da anamnese e exame físico é possível traçar os diagnósticos, estabelecer os resultados e propor as intervenções ou plano de cuidados a esses pacientes. 


\section{REFERÊNCIAS}

ALVES RN, KOVACS MJ, STALL R, PAIVA V. Fatores psicossociais e a infecção por HIV em mulheres, Maringá, PR. Rev Saude Publica; 36(Supl. 4):32-39, 2002.

BRASIL. Portaria no 1.625 de 10 de julho de 2007. Altera atribuições dos profissionais das Equipes de Saúde da Família- ESF dispostas na Política Nacional de Atenção Básica. Ministério da Saúde: Diário Oficial da União.

BRASIL. Cuidado integral as pessoas que vivem com HIV pela Atenção Básica: Manual para a equipe multiprofissional. Departamento de IST, AIDS e Hepatites virais. Brasília: Ministério da Saúde [Internet]. 2015 [cited 2017 Dec 15]; Available from: file:///C:/Users/Ju/Downloads/Cartilha___Cuidado_ Integral_as_PVHIV.pdf.

GARCIA T. R; NÓBREGA, M.M.L. Processo de enfermagem: da teoria à prática assistencial e de pesquisa. Esc. Anna Nery Rev. Enferm. [Internet]. 2009[acesso em 2021 fev 26];v.13, n.1,p:188-93. Disponível em:http://www.scielo.br/pdf/ean/v13n1/v13n1a26.pdf 3.

GLORIA M; BULECHEK; HOWARD K; BUTCHER; DOCHTERMAN, JM. Classificação das Intervenções de Enfermagem (NIC) /; [tradução Soraya Imon de Oliveira... et al]. - Rio de Janeiro: Elsevier, 2010.

HERDMAN,T.H; KAMITSURU, S; (org). Diagnósticos de Enfermagem da NANDA: definições e classificação 2015-2017-[NANDA Inernacional]. Tradução: Regina Machado Garzes. Revisão técnica: Alba Lúcia Bottura Leite de Barros [et al.]. Porto Alegre: Artmed. 2015.

HORTA W. Processo de enfermagem. São Paulo: EPU. 1979.

MATA, L. R. F.; SOUZA C. C. S.; CHIANCA T. C. M.; CARVALHO E. C.; Elaboração de diagnósticos e intervenções à luz de diferentes sistemas de classificações de enfermagem. Rev Esc Enferm USP v. 46, n.6, p:1512-18, 2012.

MARTINS, A. C. S; FERRAZ, L. M. Atuação do enfermeiro no diagnóstico e no tratamento do herpes genital, na atenção primária à saúde. Rev. APS [Internet]. 2014 [cited 28 abr 2017]; v.17, n. 2. Available from: https://aps.ufjf.emnuvens.com.br/aps/article/view/2006.

MENDES, K. D. S; SILVEIRA, R. C. C. P; GALVÃO, C. M. Revisão integrativa: método de pesquisa para a incorporação de evidências na saúde e na enfermagem. Texto Contexto Enferm. 2008;17(4):758-64.

MOORHEAD, S.; JOHNSON, M. MAAS, M.; SWANSON, E. Classificação dos resultados de enfermagem - NOC. $5^{\circ}$ ed. Rio de Janeiro: Elsevier; 2016.

NETTINA, S. M.; Práticas de Enfermagem. Rio de Janeiro: Guanabara Koogan, 2012.

NEWMAN, L.; ROWLEY, J.; HOORN SV, WIJESOORIYA NS, UNEMO, M, LOW, N.; STEVENS, G.; GOTTLIEB, S; KIARIE, J.; TEMMERMAN, M. Global estimates of the prevalence and incidence of four curable sexually transmitted infections in 2012 based on systematic review and global reporting. PLoS ONE; v.10, n.12, 2015 :e0143304.

SILVA, M. C. N. Sistematização da assistência de Enfermagem: desafio para a prática profissional. Enferm Foco. V. 8, n. 3, 2017. Doi: 10.21675/2357-707X.2017.v8.n3.1534

SOUZA T. C. F; MELO A. B; .CORRÊA, A.C; MENDONÇA ETM. Sistematização da assistência de enfermagem a um paciente portador de hiv/aids e carcinoma epidermoide: um relato de experiência. Anais 
do III Congresso de Educação em Saúde da Amazônia (COESA), Universidade Federal do Pará- 12 a 14 de novembro de 2014.

CARVALHO, K. E. G.; FREITAS, N. O.; SOUZA, J. C. et al. Teen sexual health promotion: integrative review. Journal Nursing UFPE online,v. 8, n. 7, p. 3182- 3187, 2014.

OLIVEIRA, A. S.; LIMA, T. M.; CAMPOS, F.C.; BEZERRA, K.C.; ORIÁ, M. O. B; DAMASCENO, A.K.C. Avaliação da prevalência de infecções genitais em gestantes atendidas em consulta de enfermagem ginecológica. Rev. enferm. UERJ [Internet]. Rio de Janeiro, 2013; v.21, n. 2, p: 228-33. Available from: http:// www.e-publicacoes.uerj.br/index. php/enfermagemuerj/article/view/7191.

WORLD HEALTH ORGANIZATION (WHO). Global incidence and prevalence of selected curable sexually transmitted infections - 2008. Geneva: WHO; 2012. 